\title{
Liver-Specific $\beta$-Catenin Knockout Mice Exhibit Defective Bile Acid and Cholesterol Homeostasis and Increased Susceptibility to Diet-Induced Steatohepatitis
}

\author{
Jaideep Behari, ${ }^{*}$ Tzu-Hsuan Yeh, ${ }^{*}$ \\ Lindsay Krauland, ${ }^{*}$ Wade Otruba, ${ }^{*}$ \\ Benjamin Cieply, ${ }^{\dagger}$ Beth Hauth, ${ }^{\ddagger}$ Udayan Apte, ${ }^{\dagger}$ \\ Tong Wu, ${ }^{\dagger}$ Rhobert Evans, ${ }^{\ddagger}$ \\ and Satdarshan P.S. Monga* ${ }^{\star}$ \\ From the Departments of Medicine, ${ }^{*}$ Pathology, ${ }^{\dagger}$ and \\ Epidemiology, ${ }^{\ddagger}$ University of Pittsburgh, Pittsburgh, \\ Pennsylvania
}

\begin{abstract}
Although the role of Wnt/ $\beta$-catenin signaling in liver growth and development is well established, its contribution in non-neoplastic hepatic pathologies has not been investigated. Here, we examine the role of $\beta$-catenin in a murine model of diet-induced liver injury. Mice with hepatocyte-specific $\beta$-catenin deletion (KO) and littermate controls were fed the steatogenic methionine and choline-deficient (MCD) diet or the corresponding control diet for 2 weeks and characterized for histological, biochemical, and molecular changes. KO mice developed significantly higher steatohepatitis and fibrosis on the MCD diet compared with wild-type mice. Both wild-type and KO livers accumulated triglyceride on the MCD diet but, unexpectedly, higher hepatic cholesterol levels were observed in KO livers on both control and MCD diets. Gene expression analysis showed that hepatic cholesterol accumulation in KO livers was not attributable to increased synthesis or uptake. KO mice had lower expression of bile acid synthetic enzymes but exhibited higher hepatic bile acid and serum bilirubin levels, suggesting defects in bile export. Therefore, loss of $\beta$-catenin in the liver leads to defective cholesterol and bile acid metabolism in the liver and increased susceptibility to developing steatohepatitis in the face of metabolic stress. (Am J Pathol 2010, 176:744-753; DOI: 10.2353/ajpath.2010.090667)
\end{abstract}

The Wnt/ $\beta$-catenin signal transduction pathway plays important roles in liver development, regeneration, and hepatocarcinogenesis. The pathway is regulated by diverse inputs, including the secreted Wnt ligands, 19 of which have been identified in mammals. ${ }^{1}$ The protein $\beta$-catenin is the central player in the canonical Wnt pathway. ${ }^{2,3}$ Cytoplasmic levels of $\beta$-catenin are tightly regulated. In the absence of Wnt signaling, $\beta$-catenin is bound in a multiprotein complex and is phosphorylated at specific serine and threonine residues by glycogen synthase kinase- $3 \beta$. Phosphorylated $\beta$-catenin is then targeted for destruction via ubiquitination by the $\beta$-transducing repeat-containing protein. However, in the presence of Wnt binding to the cell surface receptor family called frizzled, $\beta$-catenin remains unphosphorylated by glycogen synthase kinase- $3 \beta$ and is available for binding to the T-cell factor family of transcriptional regulators, followed by translocation to the nucleus where it transactivates several target genes. ${ }^{4-6}$ Another mechanism by which intracytoplasmic levels of $\beta$-catenin are regulated is via its association with $\mathrm{E}$-cadherin at the cell membrane, where it participates in the assembly of adherens junctions and linkage to the actin cytoskeleton. ${ }^{7}$

Adult mice with liver-specific $\beta$-catenin disruption (KO) have normal life span and only modest histological changes compared with wild-type littermates. ${ }^{8,9}$ The microsomal enzymes Cytochrome P450 2E1 (Cyp2E1) and $1 \mathrm{~A} 2$ (Cyp1A2) are down-regulated in $\beta$-catenin knockout

\footnotetext{
Supported by National Institutes of Health Institutional Training grant (T32) DK063922 (DCW) and 1K08AA017622-01 (to J.B.), and National Institutes of Health grant R01DK62277, R01CA124414, and Rango's Fund for Enhancement of Pathology Research (to S.P.S.M.).

Accepted for publication October 13, 2009.

Address reprint requests to Satdarshan (Paul) Singh Monga, M.D., Director, Division of Experimental Pathology, Associate Professor of Pathology and Medicine, University of Pittsburgh, SOM, S421-BST, 200 Lothrop Street, Pittsburgh, PA 15261. Jaideep Behari, M.D., Ph.D., Department of Medicine, Division of Gastroenterology, Hepatology, and Nutrition, 3471 Fifth Ave, Suite 916, Pittsburgh, PA 15213. E-mail: mongss@upmc.edu or beharij@upmc.edu.
} 
mice, and these mice are resistant to acetaminopheninduced liver injury. ${ }^{8,9}$ However, the effect of metabolic stressors that may cause liver injury via other mechanisms has not been tested and remains unresolved.

To investigate the effect of $\beta$-catenin on liver physiology in the face of metabolic stress, we used the methionine and choline-deficient (MCD) diet model. Mice fed the MCD diet develop steatohepatitis and fibrosis that recapitulates the histology seen in human patients with nonalcoholic steatohepatitis. ${ }^{10,11}$ Although MCD diet-fed mice do not have peripheral insulin resistance, recent reports implicate the development of hepatic insulin resistance in mice fed the MCD diet. ${ }^{2,13}$ Thus, despite differences with human nonalcoholic steatohepatitis, the MCD diet model remains an important model to study steatosis-associated liver injury.

In this report we investigated the effect of the MCD diet on liver-specific KO mice. We show that KO mice develop severe hepatic steatosis after 2 weeks on the MCD diet and exhibit defects in bile acid and cholesterol metabolism.

\section{Materials and Methods}

\section{Animals and Diets}

Liver-specific $\beta$-catenin KO mice (Ctnnb1 10xp/loxp;Allbumin$\mathrm{Cre}^{-/+}$OR Ctnnb $1^{\text {loxp/loxp}} ;$ Afp-Alb-Cre $\left.{ }^{-/+}\right)$were generated using the strategy as previously described. ${ }^{9,14}$ For this study, littermates of KO mice with wild-type Ctnnb1 alleles were used as controls and are henceforth referred to as wild-type mice. Genotypes were confirmed by PCR using specific $\beta$-catenin primers. Absence of significant $\beta$-catenin protein in the livers of KO mice was confirmed by Western blot analysis for total $\beta$-catenin in liver lysates at the end of the experiment as described below.

All experiments on mice were performed under the guidelines of the University of Pittsburgh Animal Use and Care Committee and the National Institutes of Health.

The mice were maintained under 12-hour dark-light cycles with unrestricted access to food and water. Mice were fed the MCD diet or the corresponding control diet, which was supplemented with $3 \mathrm{~g} / \mathrm{kg}$ methionine and 2 $\mathrm{g} / \mathrm{kg}$ choline (ICN Biomedicals, Costa Mesa, CA, catalog numbers 960439 and 960441, respectively). Male and female mice that were 8 to 12 weeks old at the start of the experiment were used in the study. Animals were initially fed the control diet for one week to acclimate them to the high sucrose diet, then randomly assigned to either the MCD diet or control diet groups for two additional weeks ( $N=8$ to 10 per group). At the end of the treatment period mice were sacrificed and blood and liver tissue collected for analysis.

\section{Histological and Immunohistochemical Analysis}

Liver specimens were fixed in $10 \%$ formalin and sections stained with hematoxylin and eosin (H\&E). Hepatic fibrosis was evaluated by staining sections with Masson trichrome stain and confirmed with Reticulin staining us- ing standard protocols by the University of Pittsburgh Research Histology services. An experienced pathologist (T.W.) blinded to the treatment groups evaluated the liver histology. Histological scoring was based on the Brunt classification. ${ }^{15}$ Steatosis was graded as follows: 0 (none to $5 \%$ ); 1 (5\% to $30 \%$ of hepatocytes affected); 2 (30\% to $60 \%$ of hepatocytes affected); and 3 (>60\% of hepatocytes affected). Fibrosis was determined on a scale from 0 to 4 as follows: 0 (no fibrosis); 1 (focal pericellular fibrosis in zone three); 2 (perivenular and pericellular fibrosis confined to zones two and three); 3 (bridging fibrosis); and 4 (cirrhosis). Inflammatory grade was scored on a scale from 0 to 4 as follows: 0 (no inflammation); grade 1 (sparse zone three inflammation); grade 2 (mild focal zone three hepatocyte inflammation); grade 3 (moderate zone three hepatocyte inflammation), and grade 4 (severe zone three hepatocyte inflammation). Pericellular fibrosis and balloon degeneration were scored on an arbitrary scale of 0 to 4 as follows: 0 (none), 1 (minimal, $<10 \%$ ), 2 (mild, 10 to $33 \%$ ), 3 (moderate, 33 to $66 \%$ ), and 4 (severe, $>66 \%$ ).

Oil red $\mathrm{O}$ staining for neutral lipids was performed on fresh frozen tissue cut in $8-\mu \mathrm{m}$ sections. Slides were air dried for 30 to 60 minutes and fixed in ice-cold 10\% formalin for 10 minutes. Slides were rinsed three times in distilled water, placed in propylene glycol for 5 minutes, and stained with $0.5 \%$ oil red $\mathrm{O}$ solution for 8 minutes at $60^{\circ} \mathrm{C}$. After rinsing in $85 \%$ propylene glycol solution for 30 minutes, slides were washed with distilled water and stained in Gill's hematoxylin solution for 30 seconds. After two rinses in distilled water slides were mounted with aqueous mounting medium and examined under a microscope.

Liver sections were analyzed by immunohistochemistry for $\beta$-catenin, glutamine synthetase (GS), or $\alpha$-smooth muscle actin $(\alpha$-SMA) using the indirect immunoperoxidase technique as previously described. ${ }^{9}$ Primary antibodies used for immunohistochemistry were anti- $\beta$-catenin antibody (1:200, BD Biosciences, San Jose, CA), rabbit anti-GS antibody (1:50, Santa Cruz Biotechnology, Santa Cruz, CA), and mouse anti- $\alpha$-SMA antibody (1: 500, Dako, Carpinteria, CA).

\section{Serum and Tissue Biochemical Assays}

Serum alanine aminotransferase (ALT), aspartate aminotransferase (AST), and bilirubin levels were determined by automated methods at the University of Pittsburgh Medical Center clinical chemistry laboratory. Total bile acids (TBA) in serum were measured using a commercially available kit (Diazyme, Poway, CA) as per the manufacturer's recommendations.

Lipid extraction from liver tissue was performed by chloroform/methanol extraction using a variation of the Folch method. ${ }^{16}$ Liver tissue was weighed, and $4.5 \mathrm{ml}$ of chloroform/methanol $(2: 1 \mathrm{v} / \mathrm{v})$ was added. The mixture was homogenized with a Polytron homogenizer at high speed, followed by addition of $1.5 \mathrm{ml}$ methanol and $2 \mathrm{ml}$ chloroform with mixing. The aqueous component was separated by adding $1.375 \mathrm{ml}$ of $0.73 \% \mathrm{NaCl}$ with mixing. 
The mixture was allowed to separate into two phases by centrifuging at $750 \mathrm{~g}$ for 2 minutes. The lipid-containing lower phase was collected and evaporated under nitrogen stream to obtain the lipid extract. The lipid extract was stored in 1\% Triton X-100 in chloroform until the assay was performed.

Total cholesterol was determined using an enzymatic method of Allain et al. ${ }^{17}$ This procedure involves the hydrolysis of cholesterol esters by cholesterol esterase, oxidation of cholesterol by cholesterol oxidase with formation of hydrogen peroxide, and finally a peroxidase catalyzed reaction of hydrogen peroxide with 4-aminoantipyrine and phenol to form Quinoneimine dye. The intensity of the color produced is directly proportional to the total cholesterol concentration in the sample. Duplicate samples with standards, control sera, and serum calibrators were included in each run. The coefficient of variation between runs was $1.3 \%$.

Triglycerides are determined enzymatically using the procedure of Bucolo et al. ${ }^{18}$ This procedure involves the hydrolysis of triglycerides by lipase. Glycerol is then phosphorylated by ATP and glycerol kinase to yield glycerol-1-phosphate. The Glycerol phosphate is oxidized in the presence of glycerol phosphate oxidase to produce hydrogen peroxide. The hydrogen peroxide reacts with the p-chlorophenol and 4-aminoantipyrine in the reagent to produce a Quinoneimine dye, which is read at 510/630 $\mathrm{nm}$. The intensity of the color produced is directly proportional to the total triglyceride concentration in the sample. Duplicate samples, standards, and control sera were included in each run, and the coefficient of variation between runs was $1.7 \%$. The assays were performed using an ATAC 8000 chemistry analyzer.

For hepatic TBA assay, $100 \mathrm{mg}$ frozen liver sections were minced with a razor blade and suspended in $0.5 \mathrm{ml}$ of ice-cold PBS ( $\mathrm{pH}$ 7.4) containing a protease inhibitor cocktail (Sigma-Aldrich, St. Louis, MO). Samples were homogenized and centrifuged at $600 \mathrm{~g}$ for 10 minutes at $4^{\circ} \mathrm{C}$ to remove cellular debris. The supernatant was centrifuged at $105,000 \mathrm{~g}$ and $4^{\circ} \mathrm{C}$ for 60 minutes to obtain cytoplasmic fraction. Cytoplasmic fraction TBA levels were measured using a commercially available kit (Diazyme) and normalized to protein concentration.

\section{Protein Extraction, Preparation of Hepatic Microsomes, Crude Membrane Extracts, and Western Blot Analysis}

Western blot analysis was performed as previously described. ${ }^{19}$ Mouse liver microsomes were prepared as previously described. ${ }^{9}$ Crude membrane extracts were prepared by mincing $100 \mathrm{mg}$ liver samples in $10 \mathrm{ml}$ ice-cold homogenizing buffer $(0.25 \mathrm{~mol} / \mathrm{L}$ sucrose, 10 $\mathrm{mmol} / \mathrm{L}$ Tris- $\mathrm{HCl}[\mathrm{pH}$ 7.5], containing protease inhibitor cocktail). The minced tissue was homogenized on ice in a Dounce homogenizer and filtered through a gauze sponge. The filtrate was centrifuged at $100,000 \mathrm{~g}$ for 1 hour at $4^{\circ} \mathrm{C}$ and the resulting pellet dissolved in resuspension buffer $(0.25 \mathrm{~mol} / \mathrm{L}$ sucrose, $10 \mathrm{mmol} / \mathrm{L}$ HEPES [pH 7.5], and $40 \mu \mathrm{g} / \mathrm{ml}$ PMSF). The protein concentration of each sample was determined with a Bradford protein assay kit (Sigma, St. Louis, MO).

The following primary IgG antibodies (Santa Cruz Biotechnology, Santa Cruz, CA; Cell Signaling Technology, Danvers, MA; Abcam Inc., Cambridge, MA, and Invitrogen, Carlsbad, CA) were used in this study (concentrations): mouse monoclonal anti- $\beta$-catenin (1:200); rabbit polyclonal anti-Cyp2E1 (1:200); mouse monoclonal antiactin (1:5000). Horseradish peroxidase-conjugated secondary antibodies (Chemicon International Inc., Temecula, CA) were used at concentrations of $1: 10,00$ to 1:50,000. Blots were visualized with Western Lightning chemiluminescence kit (PerkinElmer Life Sciences, Boston, MA). Membranes were stripped and reprobed with anti- $\beta$-actin or stained with Ponceau red to confirm equal loading.

\section{RNA Isolation and Real-Time PCR}

Total cellular RNA was obtained by homogenizing cells in Trizol $^{\circledR}$ reagent (Invitrogen, Carlsbad, CA). Two microgram total RNA from each sample was reverse-transcribed after DNase treatment using M-MLV reverse transcriptase (Invitrogen). Real-time PCR was performed on an ABI Prism 7000 Sequence Detection System (Applied Biosystems, Foster City, CA) using proprietary Taqman Gene Expression assays (Applied Biosystems) for the following genes: Acyl-Coenzyme A oxidase 1, palmitoyl (Acox1; Mm00443579-m1), acyl-Coenzyme A dehydrogenase, medium chain (Mcad; Mm00431611-m1), cytochrome P450, family 4 , subfamily a, polypeptide 10 (Cyp4a10; Mm01622743-g1), peroxisome proliferator activated receptor $\alpha$ (Ppar $\alpha$; Mm00440939-m1), fatty acid synthase (Fasn; Mm00662319-m1), acetyl-Coenzyme A carboxylase $\alpha$ (Accl, Mm01304289-m1), stearoylCoenzyme A desaturase 1 (Scd-1; Mm00772290-m1), sterol regulatory element binding transcription factor 1 (SREBP1; Mm01138344-m1), fatty acid binding protein 1, liver (FABP1; Mm00444340-m1), fatty acid binding protein 4, adipocyte (FABP4; Mm00445880-m1), microsomal triglyceride transfer protein (MTTP; Mm00435015-m1), sterol regulatory element binding factor 2 (Srebp2; Mm01306295-m1), nuclear receptor subfamily 1, group $\mathrm{H}$, member 3 (Nr1h3 or LXR; Mm00443454-m1), 3-hydroxy-3-methylglutaryl-Coenzyme A reductase (HMGCR; Mm01282499-m1), farnesyl diphosphate farnesyl transferase 1 (FDFT; Mm00815354-s1), ATP-binding cassette, subfamily G (WHITE), member 5 (Abcg5; Mm00446249$\mathrm{m} 1$ ), ATP-binding cassette, subfamily G (WHITE), member 8 (Abcg8; Mm00445970-m1), low-density lipoprotein receptor (Ldlr; Mm00440169-m1), cytochrome P450, family 7, subfamily a (Cyp7a1; Mm00484152-m1), cytochrome P450, family 8 , subfamily $b$, polypeptide 1 (Cyp8b1; Mm00501637-s1), cytochrome P450, family 27, subfamily a, polypeptide 1 (Cyp27; Mm00470430-m1), ATP-binding cassette subfammily $C$, member 2 (Abcc2; Mm00496899-m1), ATP-binding cassette subfamily B, member 11 (Abcb11; Mm00445168-m1), and heme oxygenase 1 (Hmox1; Mm00516004-m1). The Relative expression levels were calculated after normalization to 
$\beta$-actin (Applied Biosystems, catalog no. 4352341E) using the $\Delta \Delta$-Ct method recommended by Applied Biosystems (User Bulletin no. 2, 1997).

\section{Statistical Analysis}

Results are expressed as mean \pm SEM for each group. The number of samples included in each experiment is indicated in the figure legends. Data were analyzed using one-way analysis of variance, followed by pairwise comparisons with the Student $t$ test (two-tailed). Values of $P<$ 0.05 were considered significant and are presented in the results section.

\section{Results}

Liver-Specific $\beta$-Catenin Knockout Mice Develop Severe Steatohepatitis on the MCD Diet

Wild-type mice fed the control diet had normal liver morphology and no steatosis (Figure 1A). After 2 weeks on the MCD diet, livers from wild-type mice appeared grossly normal but on histological examination had mild steatosis and inflammation. In contrast, knockout mice on the control diet on gross examination appeared steatotic and on histological examination had mild steatohepatitis that was comparable with the wild-type mice on the MCD diet. Knockout mice on the MCD diet were also grossly steatotic. All mice in this group developed striking predominantly macrovesicular steatosis involving $80 \%$ to $100 \%$ of the liver parenchyma.

Both strains of mice on the MCD diet had significantly higher inflammation than mice on the control diet (Figure $1 \mathrm{~B}$ ). There was a trend toward higher inflammation in the knockout mice on the MCD diet compared with wild-type mice on this diet but did not reach statistical significance. Hepatocyte balloon degeneration was significantly higher in knockout mice on both the control and MCD diets compared with their wild-type littermates (Figure 1B).

\section{Knockout Mice on the MCD Diet Have Increased Hepatic Injury and Fibrosis}

To assess hepatic injury, serum ALT and AST levels were evaluated in control and experimental groups (Figure 2A). ALT levels were significantly higher in the two strains of mice on the MCD diet compared with the control diet groups. On the control diet, KO had higher ALT level than wild-type mice. No significant difference was found in serum AST levels.

To evaluate hepatic fibrosis, liver sections were stained with Masson trichrome stain and Reticulin stain (Figure 2B, top two panels). No fibrosis was seen in wild-type mice on the control diet, whereas mildly increased but statistically insignificant fibrosis was noted in wild-type mice on the MCD diet and KO mice on the control diet. In contrast, KO mice on the MCD diet had

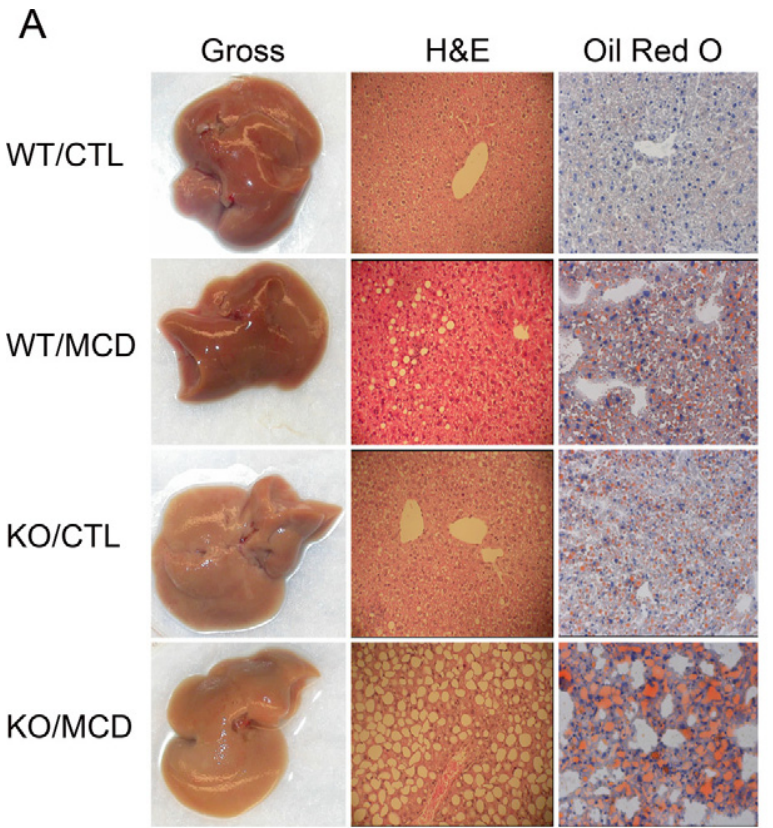

B
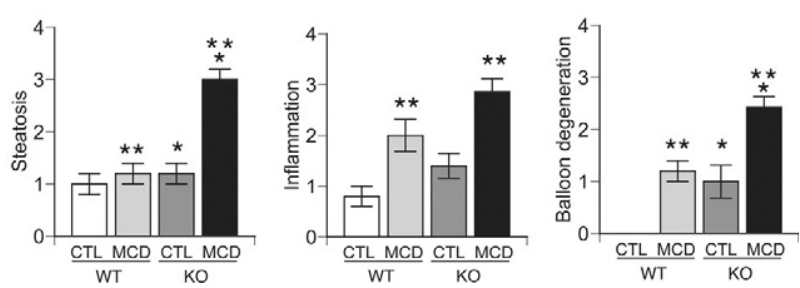

Figure 1. $\beta$-Catenin knockout mice fed the MCD diet develop severe steatohepatitis. A: Gross, H\&E-, and oil red O-stained sections of livers from wild-type or KO mice fed either the control or MCD diets as indicated (liver sections at $\times 200$ magnification). B: Quantification of steatosis, inflammation, and hepatocyte ballooning seen in the H\&E stained sections by a pathologist blinded to the treatment groups. Values are expressed as means \pm SEM from five to seven animals per group. ${ }^{*} P<0.05$ wild-type versus $\mathrm{KO}$ groups, ${ }^{* *} P<$ 0.05 CTL versus MCD groups. WT/CTL indicates wild-type mice on control diet; WT/MCD, wild-type mice on MCD diet; KO/CTL, KO mice on the control diet; $\mathrm{KO} / \mathrm{MCD}$, KO mice on MCD diet.

significantly higher fibrosis and pericellular fibrosis (Figure 2, B and C).

Immunohistochemistry for $\alpha$-SMA, a marker of activated stellate cells, was performed and consistent with the results seen with trichrome and reticulin staining revealed increased $\alpha$-SMA staining in KO mice on the MCD diet (Figure 2B, bottom panel). Minimal $\alpha$-SMA staining was seen in wild-type mice on MCD diet and in KO mice on the control diet.

\section{Levels of $\beta$-Catenin do not Change Significantly on the MCD Diet}

Because most $\beta$-catenin in hepatocytes is observed at the membrane bound to E-cadherin, we performed immunohistochemical staining for $\beta$-catenin on liver sections from wild-type mice on the two diets. No significant difference in cellular localization or intensity of staining of $\beta$-catenin was found between the control or MCD diet-fed groups (Figure 3A, upper panel). Immunoprecipitation 
A

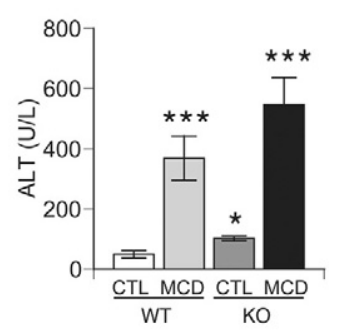

B

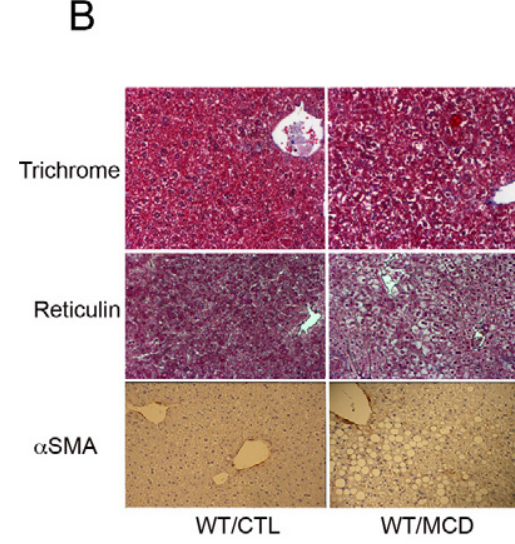

C

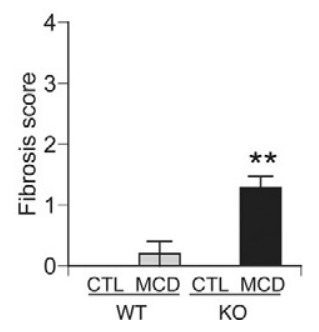

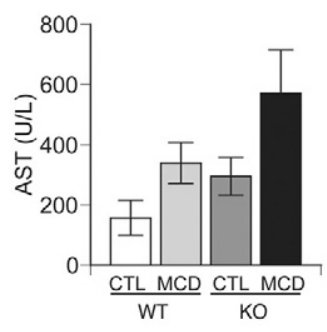
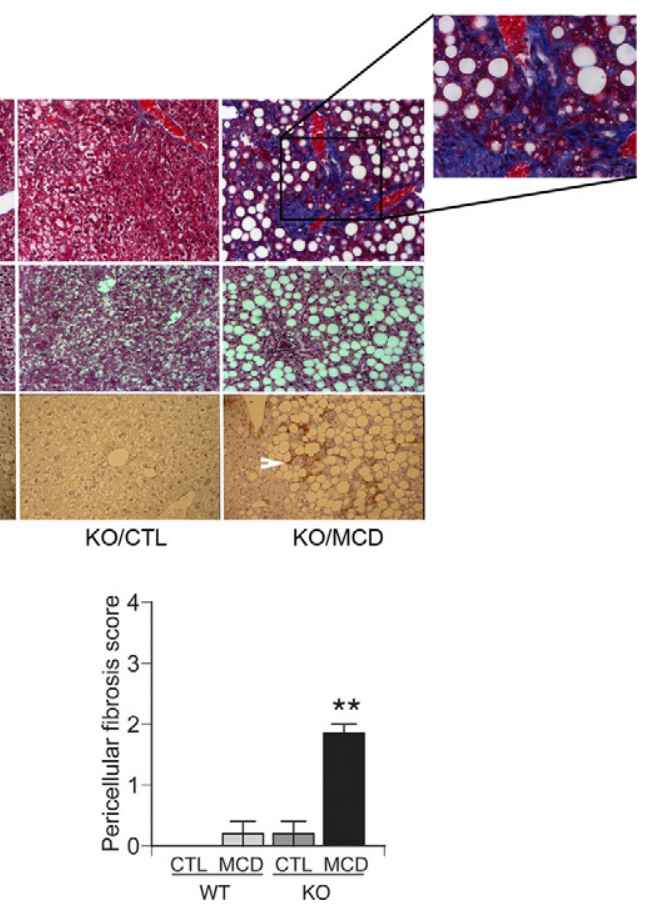

with $\beta$-catenin and immunoblotting with $\mathrm{E}$-cadherin antibody revealed no significant difference in $\beta$-catenin-Ecadherin interaction between the two groups (data not shown). As expected, staining for $\beta$-catenin was negative in both groups of $\mathrm{KO}$ animals.

GS is a downstream target of $\beta$-catenin and is expressed strongly in zone 3 hepatocytes. Immunohistochemical staining for GS revealed almost complete absence of staining from both groups of $\mathrm{KO}$ mice, con- sistent with the loss of $\beta$-catenin in these animals (Figure $3 \mathrm{~A}$, bottom panel). In wild-type mice, prominent zone 3 GS staining was seen that was not significantly different between the two diet groups.

To confirm these results, Western blot analysis for total $\beta$-catenin on liver lysates was performed. No significant difference in the levels of total $\beta$-catenin was seen in wild-type mice on the control or MCD diets (Figure 3B). As expected, $\beta$-catenin protein levels were significantly

A

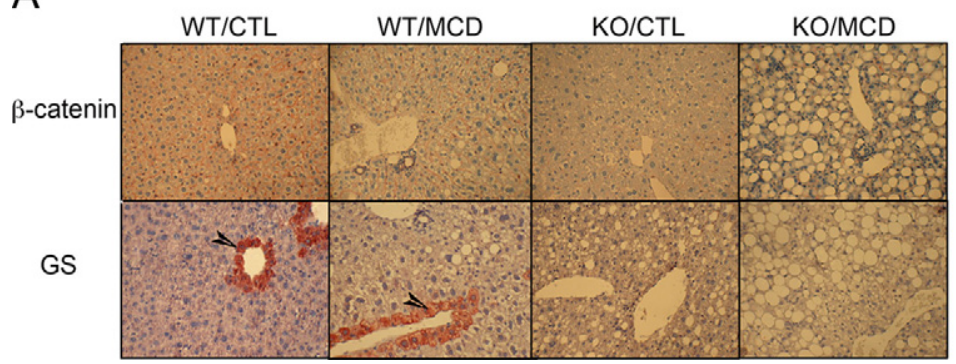

B

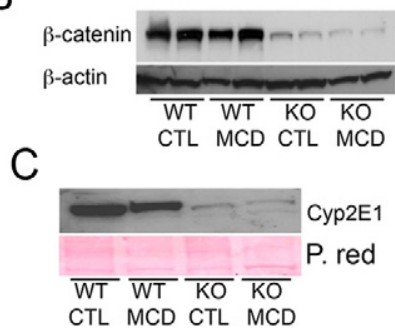

Figure 3. Levels of $\beta$-catenin and its target genes in knockout and wild-type animals fed the control or MCD diets. A, Upper panel: Immunohistochemical staining with total $\beta$-catenin. Brown staining for $\beta$-catenin is visible in the two wild-type groups at the cell membrane. No staining is seen in the two KO groups. A, Lower panel: Glutamine synthetase staining. Brown glutamine synthetase staining is visible in a narrow rim of perivenous hepatocytes from wild-type animals fed either the control or MCD diets (black arrowhead). No staining is visible in KO livers (All images are at $\times 200$ magnification). B: Western blot analysis for total $\beta$-catenin using liver lysates from the four experimental groups. Two representative samples from each experimental group are shown. The membrane was stripped and reprobed with $\beta$-actin as internal loading control. C: Western blot analysis for Cyp2E1 using liver microsomal preparation from each of the experimental groups. One representative sample from each group is shown. The membrane was stained with Ponceau red to confirm equal loading. WT/CTL indicates wild-type mice on control diet; WT/MCD, wild-type mice on MCD diet; KO/CTL, knockout mice on the control diet; KO/MCD, knockout mice on MCD diet. 
A
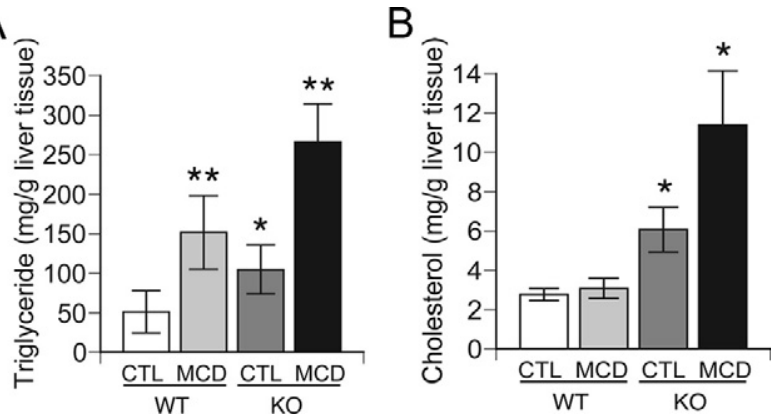

Figure 4. Hepatic triglyceride and cholesterol levels in control-diet and MCD-diet fed wild-type and KO mice. A: Hepatic triglyceride levels B: Hepatic cholesterol levels. Results are expressed as means \pm SEM of five samples per group. ${ }^{*} P<0.05$ wild-type versus KO groups, ${ }^{*} P<0.05 \mathrm{CTL}$ versus MCD groups. WT/CTL indicates wild-type mice on control diet WT/MCD, wild-type mice on MCD diet; KO/CTL, knockout mice on the control diet; KO/MCD, knockout mice on MCD diet.

lower in $\mathrm{KO}$ animals. A faint band corresponding to $\beta$-catenin from nonparenchymal cell source was noted in the two KO groups.

Cyp2E1, a downstream target of $\beta$-catenin, plays a role in liver injury in the setting of steatohepatitis, by increasing oxidative stress. Thus, the result that loss of $\beta$-catenin resulted in increased liver injury on the MCD diet led us to evaluate the levels of microsomal Cyp2E1 levels. Cyp2E1 was significantly lower in $\mathrm{KO}$ mice compared with wildtype animals, which was consistent with our previous results. ${ }^{9}$ We also did not observe any consistent differences in Cyp2E1 levels in wild-type mice on the control or MCD diets after 2 weeks (Figure 3C). We conclude from these results that significantly greater steatohepatitis occurs in the $\beta$-catenin KO mice, despite loss of Cyp2E1 expression.

\section{$\beta$-Catenin KO Mice Have Hepatic Cholesterol Accumulation}

To further evaluate the steatosis noted in $\mathrm{KO}$ mice on histological examination, hepatic triglyceride quantification was performed. As expected, both $\mathrm{KO}$ and wild-type mice on the MCD diet had significantly higher liver triglyceride levels compared with the two groups of mice on the control diet. Whereas KO mice on the control diet had significantly higher hepatic triglyceride compared with wild-type mice on the control diet, no significant difference was found between wild-type and $\mathrm{KO}$ mice on the MCD diet (Figure 4A).

Given the striking difference in liver histology between the $\mathrm{KO}$ and wild-type mice on the MCD diet, the modest difference in their hepatic triglyceride levels was surprising and did not explain the dramatic phenotype. Therefore, we quantified hepatic total cholesterol levels in the four groups of mice. In wild-type animals, no difference in hepatic cholesterol levels was found between the control and MCD diet-fed groups. However, both control and MCD diet fed KO animals had significantly higher hepatic cholesterol levels compared with wild-type animals (Figure $4 \mathrm{~B}$ ). MCD diet-fed KO mice displayed a trend toward

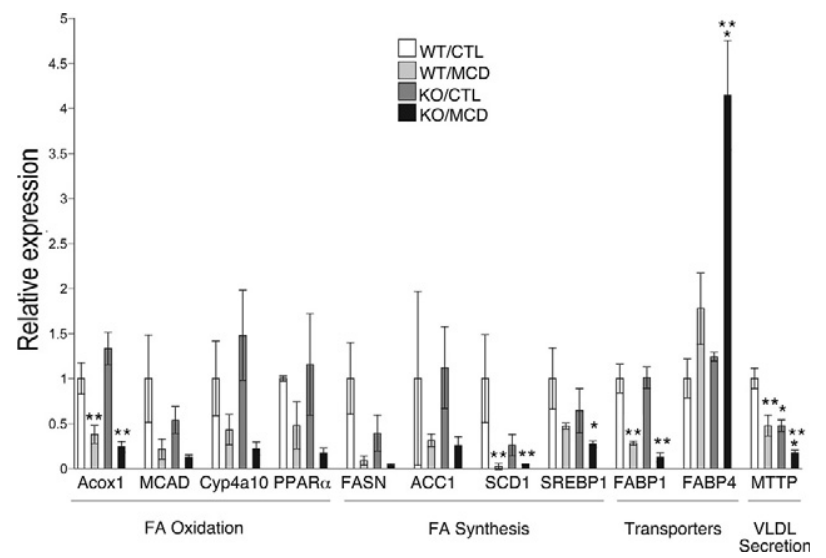

Figure 5. Expression analysis by quantitative real-time PCR of genes involved in hepatic fatty acid and triglyceride metabolism. Results are expressed as means \pm SEM of five to seven samples per group, with each sample analyzed in duplicate. ${ }^{*} P<0.05$ wild-type versus $\mathrm{KO}$ groups, ${ }^{* *} P<$ 0.05 CTL versus MCD groups. WT/CTL indicates wild-type mice on control diet; WT/MCD, wild-type mice on MCD diet; KO/CTL, knockout mice on the control diet; KO/MCD, knockout mice on MCD diet.

higher hepatic cholesterol compared with the control diet-fed KO mice but missed statistical significance.

\section{Evaluation of Expression of Genes Involved in Hepatic Fatty Acid and Triglyceride Metabolism}

We first evaluated expression of several genes involved in fatty acid oxidation, fatty acid biosynthesis, fatty acid transport, and very low-density lipoprotein secretion (Figure 5). Expression of Acox1 was significantly lower in both MCD fed groups of mice but not significantly different between $\mathrm{KO}$ or wild-type mice. No significant difference was found in the expression of MCAD (encoded by Acadm), Cyp4a10, or peroxisome proliferator-activated receptor $\alpha$.

Expression of genes involved in fatty acid biosynthesis showed a trend toward decreased expression of Fasn and Accl but did not reach statistical significance. As reported previously by Rizki et al, expression of SCD1 was almost completely absent in the two groups of MCD diet-fed animals. ${ }^{20}$ The expression of Srebp1, the regulator of fatty acid synthesis, was lower in the two MCD diet-fed groups, but reached statistical significance only in $\mathrm{KO}$ mice.

Differential expression of two fatty acid transporters was noted. In the case of Fabp1, expression was significantly lower in both MCD diet groups. However, expression of Fabp4 was higher in the MCD groups and was statistically significant in the KO group on MCD diet.

We then examined the expression of MTTP, which is critical for the assembly and secretion of very low-density lipoprotein particles from the liver. In wild-type animals, expression of MTTP was significantly lower on the MCD diet than on the control diet. In the KO mice on the control diet, MTTP expression was twofold lower than their wild-type littermates on the same diet. Similarly, on the MCD diet, KO animals had significantly lower MTTP expression compared with the other three groups of mice. 
A

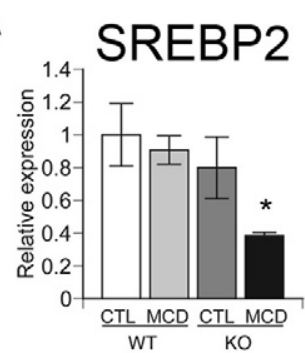

B

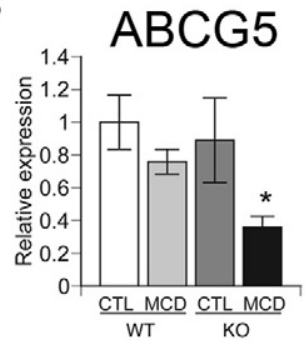

C

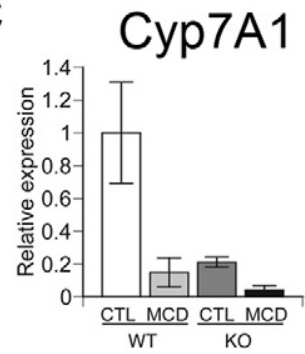

LXR

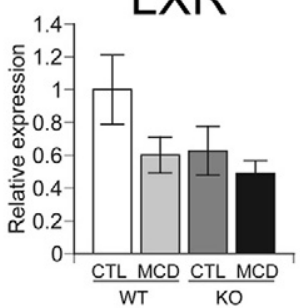

ABCG8

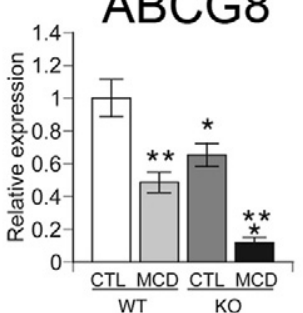

Сyp8b1

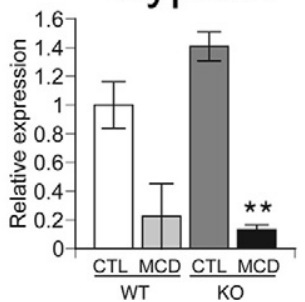

HMGCR

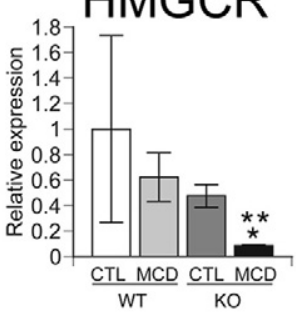

LDLR

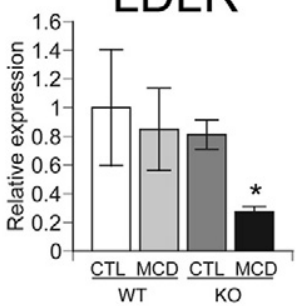

Сyp27

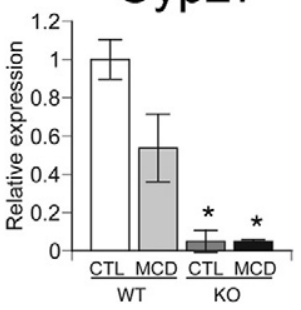

Figure 6. Expression analysis by quantitative real-time PCR of genes involved in hepatic cholesterol metabolism. A: Genes involved in cholesterol biosynthesis. B: Genes involved in cholesterol efflux and transport. C: Genes encoding the cytochrome P450 enzymes involved in the metabolism of cholesterol to bile acids. Results are expressed as means \pm SEM of five to seven samples per group, with each sample analyzed in duplicate. ${ }^{*} P<0.05$ wild-type versus KO groups, ${ }^{* * *} P<$ 0.05 CTL versus MCD groups. WT/CTL indicates wild-type mice on control diet; WT/MCD, wild-type mice on MCD diet; KO/CTL, knockout mice on the control diet; KO/MCD, knockout mice on MCD diet.

\section{Evaluation of Expression of Genes Involved in Cholesterol Metabolism}

Hepatic cholesterol accumulation in control and MCD diet-fed $\mathrm{KO}$ mice was unexpected because chow-fed $\mathrm{KO}$ mice have normal cholesterol levels in the liver (our unpublished results). Evaluation of expression of genes involved in cholesterol biosynthesis (Figure 6A) showed significantly lower expression of HMGCR, the rate-limiting enzyme in cholesterol biosynthesis, in $\mathrm{KO}$ mice on the MCD diet. Consistent with this result, expression of Srebp2, a gene important in regulating cholesterol biosynthesis, was also significantly lower in this group. The nuclear receptor $\mathrm{Nr} 1 \mathrm{~h} 3(\mathrm{LXR})$ is an important mediator of cholesterol homeostasis and regulates Cyp7a1 expression and bile acid biosynthesis from cholesterol. We found no significant difference in LXR expression between wild-type and $\mathrm{KO}$ animals on either experimental diet.

We then examined the expression of Abcg5 and Abcg8, important cholesterol efflux transporters (Figure $6 \mathrm{~B})$. In the case of Abcg5, MCD diet-fed KO mice had significantly lower expression compared with wild-type mice, whereas expression of Abcg8 was lower in both groups of $\mathrm{KO}$ mice compared with the wild-type mice on the corresponding diet. Similarly, expression of the hepatic LDL receptor was lower in the $\mathrm{KO}$ animals on MCD diet.

We next evaluated the expression of Cyp7a1, Cyp8b1, and Cyp27, which are cytochrome P450 enzymes important in bile acid synthesis from cholesterol (Figure 6C). Expression of Cyp7a1 was consistently lower in $\mathrm{KO}$ mice and MCD-fed wild-type mice, but the difference narrowly missed statistical significance because of variation in the expression of Cyp7a1 in control diet-fed wild-type mice. Expression of Cyp8b1 was lower in MCD-fed wild-type and $\mathrm{KO}$ as compared with the respective control diets. Expression of Cyp27, however, was significantly lower in both control diet and MCD diet-fed KO mice compared with the corresponding wild-type mice.

\section{Increased Hepatic Bile Acid and Serum Bilirubin Levels in $\beta$-Catenin KO Mice}

Gene expression analysis with bile acid biosynthetic enzymes presented above suggested that cholesterol accumulation in $\mathrm{KO}$ mice might result from decreased conversion to bile acids. One explanation for the low expression of bile acid biosynthetic enzymes in $\mathrm{KO}$ mice could be a primary defect in expression of bile acid synthetic enzymes. A second possibility could be that $\mathrm{KO}$ 
A

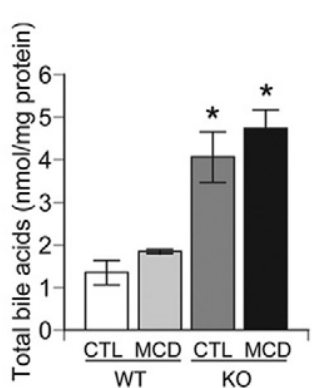

C

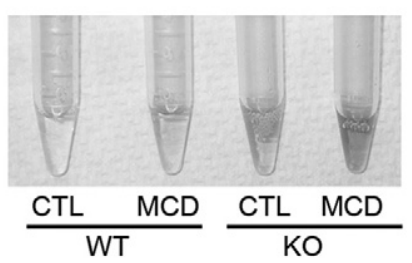

B

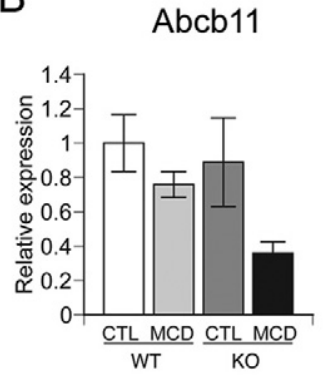

D

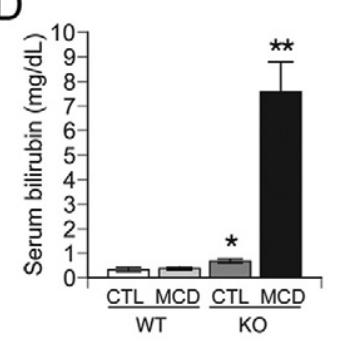

Abcc2

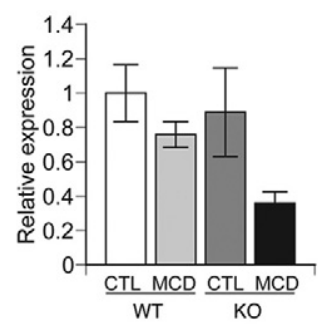

E

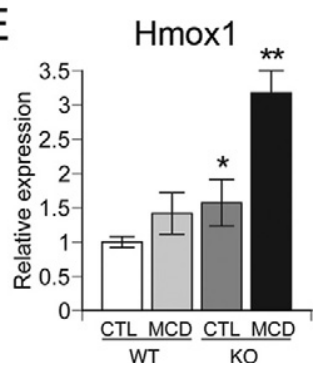

Figure 7. Analysis of hepatic bile acids and serum bilirubin levels. A: Hepatic total bile acid levels in wild-type and $\mathrm{KO}$ mice on control or MCD diet. B: Analysis of expression levels by real-time PCR of genes involved in export of bile acids and bilirubin. Data are expressed as arbitrary units relative to the expression level in the WT/CTL group. C: Representative serum samples from each treatment group. D: Serum total bilirubin levels. E: Analysis of expression levels of Hmox1 by real-time PCR. Results are expressed as means \pm SEM of 5 samples per group, with each sample analyzed in duplicate. ${ }^{* * *} P<0.01$ compared with all other groups, ${ }^{*} P<$ 0.05 wild-type versus KO groups. WT/CTL indicates wild-type mice on control diet; WT/MCD, wild-type mice on MCD diet; KO/CTL, knockout mice on the control diet; KO/MCD, knockout mice on MCD diet. mice have a bile secretory defect that results in elevated hepatic bile acid levels and negative feedback inhibition of bile acid biosynthetic enzymes. To distinguish between these two possibilities, we assayed total bile acid levels in livers from the four groups of mice. In support of the latter hypothesis, KO mice on both control and MCD diets had twofold higher hepatic TBA levels compared with wild-type mice (Figure 7A). To test whether there were differences in expression of the major bile acid transporters in $\mathrm{KO}$ mice, we performed quantitative realtime PCR for Abcb11 (Bsep) and Abcc2 (Mrp2). No significant difference was found in the expression of these transporters between wild-type and $\mathrm{KO}$ animals on either diet (Figure 7B).

During collection of serum samples, $\mathrm{KO} / \mathrm{MCD}$ mice were noted to have intense yellow coloration suggestive of jaundice (Figure 7C). Serum bilirubin assays revealed that $\mathrm{KO} / \mathrm{control}$ mice had twofold higher serum total bilirubin level (Figure 7D). Although within normal limits, the bilirubin levels in the $\mathrm{KO} / \mathrm{control}$ group were consistently and significantly higher than in the wild-type/control group. On the MCD diet, KO mice had approximately 15-fold higher serum bilirubin level compared with both groups of wild-type mice and seven- to eightfold higher level compared with the $\mathrm{KO} / \mathrm{control}$ group. In $\mathrm{KO}$ mice on both control and MCD diets, more than $90 \%$ of serum bilirubin was conjugated (data not shown).

To explain the higher serum bilirubin levels in $\mathrm{KO}$ mice, we hypothesized that $\mathrm{KO}$ mice have increased hepatic expression of Hmox1. This gene encodes the enzyme heme oxygenase 1, an inducible enzyme that catalyzes the rate-limiting step in the conversion of heme to biliverdin, which is then reduced to bilirubin. Both biliverdin and bilirubin have strong antioxidative properties and Hmox1 expression has been shown to be upregulated with oxidative stress. ${ }^{21}$ Consistent with this hypothesis, we saw significantly higher expression of $\mathrm{Hmox} 1$ in both $\mathrm{KO}$ groups relative to the corresponding wild-type mice (Figure $7 \mathrm{E})$.

\section{Discussion}

$\beta$-Catenin plays a critical role in liver development, and its prenatal loss in the liver is lethal. ${ }^{22}$ Yet loss of $\beta$-catenin in the adult mouse liver results in a surprisingly mild phenotype and no difference in normal lifespan, with changes in serum biochemistry reminiscent of mild hepatic injury especially in the older animals. ${ }^{8,9}$ Furthermore, liver-specific KO mice are resistant to liver injury induced by acetaminophen overdose that is consistent with the low levels of hepatic microsomal Cyp2E1 levels seen in these mice. ${ }^{823}$ Our laboratory has recently also shown that inducing the low hepatic microsomal levels of Cyp2E1 in $\beta$-catenin $\mathrm{KO}$ mice by pyrazole treatment partially restores the susceptibility of $\mathrm{KO}$ mice to acetaminophen-induced hepatotoxicity, underscoring the functional significance of low Cyp2E1 levels in $\mathrm{KO}$ mice. ${ }^{23}$ Because Cyp2E1 has been implicated in the pathogenesis of steatohepatitis we asked whether $\beta$-catenin $\mathrm{KO}$ mice would be protected against hepatic steatosis and injury because of their low hepatic levels of Cyp2E1. ${ }^{24,25}$ We used the MCD diet model of experimental steatohepatitis to test this hypothesis because this diet has been shown to induce Cyp2E1 levels. ${ }^{26}$

The striking increase in steatophepatitis in $\mathrm{KO}$ livers on the MCD diet after only two weeks of exposure to the diet suggested that $\beta$-catenin confers a protective effect on the liver in the face of metabolic stress. Hepatic fibrosis attributable to hepatic stellate cell activation is a wellrecognized end result of injury occurring from a wide variety of etiologies in the liver. ${ }^{27,28}$ Prior studies have shown increased fibrosis on the MCD diet, with more advanced fibrosis correlating with greater steatohepatitis in several experimental models. ${ }^{29-31}$ The significantly greater liver fibrosis and pericellular fibrosis in $\mathrm{KO}$ mice on the MCD diet, which correlates with the greater necroinflammatory activity seen in the $\mathrm{KO}$ mice, is consistent with these prior reports. 
Based on the histological findings, we expected to find higher liver triglyceride levels in KO mice. Surprisingly, only modest differences in hepatic triglycerides levels and expression of fatty acid metabolic genes were found between $\mathrm{KO}$ and wild-type mice. The subsequent finding that KO mice had hepatic cholesterol accumulation was unexpected and unlikely to be attributable to the MCD diet, because KO mice on the control diet also had higher hepatic cholesterol levels whereas the wild-type mice on the MCD diet did not.

Gene expression analysis showed that genes involved in cholesterol biosynthesis and uptake were down-regulated in KO mice, suggesting that hepatic cholesterol accumulation was not attributable to increased synthesis or uptake. Cholesterol excretion from the liver via bile, directly or via conversion to bile acids, is critical for cholesterol homeostasis. A defect in bile export defect would be expected to lead to hepatic cholesterol accumulation. Our finding that $\mathrm{KO}$ livers have higher hepatic bile acid concentration supports the hypothesis that $\mathrm{KO}$ mice accumulate cholesterol because of the bile secretory defect. Indeed, we have recently found that KO mice have defects in bile acid homeostasis and lower bile flow rate (manuscript in preparation). Because $\mathrm{KO}$ mice on chow diet have normal hepatic cholesterol levels (our unpublished results), we speculate that in response to the higher bile acid levels in the liver KO mice on chow diet are able to suppress de novo cholesterol synthesis sufficiently to maintain normal cholesterol levels in the liver. However, the higher fat content of the MCD and control diets may cause sufficient upregulation of cholesterol biosynthesis to induce hepatic cholesterol accumulation in $\mathrm{KO}$ mice. Hepatic bile acid accumulation in $\mathrm{KO}$ livers could account for the increased oxidative stress. Increased expression of $\mathrm{Hmox} 1$ as a protective response to oxidative stress combined with the defect in bile export would account for the jaundice found in KO mice, an effect that is worsened by the metabolic stress imposed by the MCD diet.

Our findings have potential clinical implications. Because $\beta$-catenin has been implicated in the pathogenesis of a significant percentage of hepatocellular carcinomas, targeted therapy against $\beta$-catenin is an attractive strategy in the treatment of these cancers. Our findings also suggest that $\beta$-catenin is an additional player in the complex network of nuclear receptors that regulates bile acid and lipid metabolism in the liver. Identifying the interactions between these receptors and $\beta$-catenin may help elucidate additional regulatory mechanisms that could have potential therapeutic implications in both cholestatic and metabolic liver diseases.

In conclusion, we report here that liver-specific $\beta$-catenin KO mice exhibit cholestasis, and feeding the steatogenic MCD diet leads to severe macrovesicular steatosis, hepatic cholesterol accumulation, and jaundice. In addition to multifaceted roles in liver development, regeneration, and hepatocarcinogenesis, our results point toward an important role of $\beta$-catenin in hepatic bile acid and cholesterol homeostasis, and in protecting the adult liver against metabolic stress.

\section{References}

1. Miller JR: The Wnts. Genome Biol 2002, 3:REVIEWS3001

2. Nelson WJ, Nusse R: Convergence of Wnt, beta-catenin, and cadherin pathways. Science 2004, 303:1483-1487

3. Willert K, Nusse R: Beta-catenin: a key mediator of Wnt signaling. Curr Opin Genet Dev 1998, 8:95-102

4. Barker N: The canonical Wnt/beta-catenin signalling pathway. Methods Mol Biol 2008, 468:5-15

5. Brantjes H, Barker N, van Es J, Clevers H: TCF: lady Justice casting the final verdict on the outcome of Wnt signalling. Biol Chem 2002, 383:255-261

6. Moon RT: Wnt/beta-catenin pathway. Sci STKE 2005, 2005:cm1

7. Hartsock A, Nelson WJ: Adherens and tight junctions: structure, function and connections to the actin cytoskeleton. Biochim Biophys Acta 2008, 1778:660-669

8. Sekine S, Lan BY, Bedolli M, Feng S, Hebrok M: Liver-specific loss of beta-catenin blocks glutamine synthesis pathway activity and cytochrome p450 expression in mice. Hepatology 2006, 43:817-825

9. Tan X, Behari J, Cieply B, Michalopoulos GK, Monga SP: Conditional deletion of beta-catenin reveals its role in liver growth and regeneration. Gastroenterology 2006, 131:1561-1572

10. Koteish A, Diehl AM: Animal models of steatosis. Semin Liver Dis 2001, 21:89-104

11. Leclercq IA, Farrell GC, Field J, Bell DR, Gonzalez FJ, Robertson GR: CYP2E1 and CYP4A as microsomal catalysts of lipid peroxides in murine nonalcoholic steatohepatitis. J Clin Invest 2000, 105:1067-1075

12. Leclercq IA, Lebrun VA, Starkel P, Horsmans YJ: Intrahepatic insulin resistance in a murine model of steatohepatitis: effect of PPARgamma agonist pioglitazone. Lab Invest 2007, 87:56-65

13. Rinella ME, Green RM: The methionine-choline deficient dietary model of steatohepatitis does not exhibit insulin resistance. J Hepatol 2004, 40:47-51

14. Apte U, Zeng G, Thompson MD, Muller P, Micsenyi A, Cieply B, Kaestner $\mathrm{KH}$, Monga SP: beta-Catenin is critical for early postnatal liver growth. Am J Physiol Gastrointest Liver Physiol 2007, 292 : G1578-G1585

15. Brunt EM, Janney CG, Di Bisceglie AM, Neuschwander-Tetri BA, Bacon BR: Nonalcoholic steatohepatitis: a proposal for grading and staging the histological lesions. Am J Gastroenterol 1999, 94:2467-2474

16. Folch J, Ascoli I, Lees M, Meath JA, Le BN: Preparation of lipide extracts from brain tissue. J Biol Chem 1951, 191:833-841

17. Allain CC, Poon LS, Chan CS, Richmond W, Fu PC: Enzymatic determination of total serum cholesterol. Clin Chem 1974, 20:470-475

18. Bucolo G, David H: Quantitative determination of serum triglycerides by the use of enzymes. Clin Chem 1973, 19:476-482

19. Micsenyi A, Tan X, Sneddon T, Luo JH, Michalopoulos GK, Monga SP: Beta-catenin is temporally regulated during normal liver development. Gastroenterology 2004, 126:1134-1146

20. Rizki G, Arnaboldi L, Gabrielli B, Yan J, Lee GS, Ng RK, Turner SM, Badger TM, Pitas RE, Maher JJ: Mice fed a lipogenic methioninecholine-deficient diet develop hypermetabolism coincident with hepatic suppression of SCD-1. J Lipid Res 2006, 47:2280-2290

21. Maines MD: The heme oxygenase system: a regulator of second messenger gases. Annu Rev Pharmacol Toxicol 1997, 37:517-554

22. Tan X, Yuan Y, Zeng G, Apte U, Thompson MD, Cieply B, Stolz DB Michalopoulos GK, Kaestner KH, Monga SP: Beta-catenin deletion in hepatoblasts disrupts hepatic morphogenesis and survival during mouse development. Hepatology 2008, 47:1667-1679

23. Apte U, Singh S, Zeng G, Cieply B, Virji MA, Wu T, Monga SP Beta-catenin activation promotes liver regeneration after acetaminophen-induced injury. Am J Pathol 2009, 175:1056-1065

24. Chalasani N, Gorski JC, Asghar MS, Asghar A, Foresman B, Hall SD, Crabb DW: Hepatic cytochrome P450 2E1 activity in nondiabetic patients with nonalcoholic steatohepatitis. Hepatology 2003, 37:544-550

25. Weltman MD, Farrell GC, Hall P, Ingelman-Sundberg M, Liddle C Hepatic cytochrome P450 2E1 is increased in patients with nonalcoholic steatohepatitis. Hepatology 1998, 27:128-133

26. Weltman MD, Farrell GC, Liddle C: Increased hepatocyte CYP2E1 expression in a rat nutritional model of hepatic steatosis with inflammation. Gastroenterology 1996, 111:1645-1653

27. Bataller R, Brenner DA: Liver fibrosis. J Clin Invest 2005, 115:209-218

28. Friedman SL: Mechanisms of disease: mechanisms of hepatic fibro- 
sis and therapeutic implications. Nat Clin Pract Gastroenterol Hepatol 2004, 1:98-105

29. dela Pena A, Leclercq IA, Williams J, Farrell GC: NADPH oxidase is not an essential mediator of oxidative stress or liver injury in murine MCD diet-induced steatohepatitis. J Hepatol 2007, 46:304-313

30. Ota T, Takamura T, Kurita S, Matsuzawa N, Kita Y, Uno M, Akahori H, Misu H, Sakurai M, Zen Y, Nakanuma Y, Kaneko S: Insulin resistance accelerates a dietary rat model of nonalcoholic steatohepatitis. Gastroenterology 2007, 132:282-293

31. Yamaguchi K, Yang L, McCall S, Huang J, Yu XX, Pandey SK, Bhanot $S$, Monia BP, Li YX, Diehl AM: Inhibiting triglyceride synthesis improves hepatic steatosis but exacerbates liver damage and fibrosis in obese mice with nonalcoholic steatohepatitis. Hepatology 2007 , 45:1366-1374 